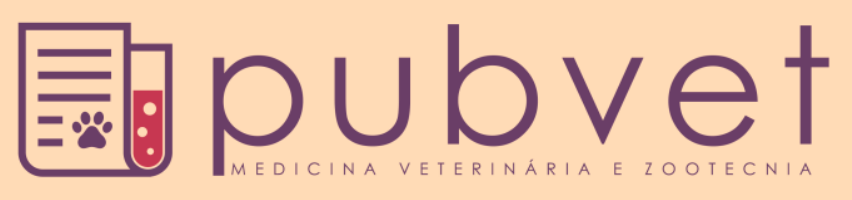

ISSN $1982-1263$

https://doi.org/10.22256/pubvet.v12n3a42.1-9

\title{
Checklist dos parasitos do peixe Lua (Mola mola: Molidae) no mundo
}

\author{
Naibe Cristina de Figueiredo ${ }^{\bullet} 1^{*}$, José Ticiano Arruda Ximenes de Lima ${ }^{\bullet}{ }^{2}$, Carlos Iberê \\ Freitas ${ }^{2}$, Claudio Giovanio da Silva 2
}

${ }^{1}$ Programa de Pós-Graduação em Produção Animal, Universidade Federal Rural do Semi-Árido, Av. Francisco Mota, 572, Costa e Silva, 59625-900, Mossoró, Rio Grande do Norte, Brasil. *Autor para correspondência, E-mail: naibecristina@hotmail.com

${ }^{2}$ Universidade Federal Rural do Semi-Árido, Av. Francisco Mota, 572, Costa e Silva, 59625-900, Mossoró, Rio Grande do Norte, Brasil.

\begin{abstract}
RESUMO. Devido à escassez de trabalhos relacionados ao parasitismo no mundo, sendo assim, percebe-se a importância de realizar uma revisão bibliográfica em artigos científicos, dissertações e teses em bancos de dados e periódicos de fácil acesso para os estudantes e pesquisadores acerca das ocorrências de parasitas em $M$. mola entre os anos de 1904 - 2017. A espécie de peixe conhecida popularmente como peixe lua (Mola mola: Molidae) são encontrados em todos os oceanos do mundo. O parasitismo pode ser uma das possíveis causas de encalhe desta família no Brasil, uma vez que existe um número de publicações cientificas reduzida de relatos de ocorrência da espécie M. mola. Foram encontrados 51 trabalhos relacionados a parasitos da espécie Mola mola no mundo, no estudo foram registradas 44 espécies de parasitos e 9 espécies são sinônimos, ou seja, são parasitos conhecidos por mais de um nome. $\mathrm{O}$ estudos possibilitou encontrar a ocorrência de parasitária em M. mola nos cinco filos e nos cinco continentes, destes houve maior registro de ocorrência parasitária na Europa nos filos Platylhelminte $(n=28)$, Arthopoda $(n=15)$ e Nematoda $(n=1)$ e na América os filos Acanthocephala $(n=1)$, Platylhelmintes $(n=27)$ e Arthopoda $(n=15)$, Oceania Platylhelmintes $(n=7)$, Nematoda $(n=1)$ e Arthopoda $(n=14)$, na Ásia foram registrados os filos Platylhelmintes $(n=3)$ e Arthopoda $(n=5)$, na África foram registrados estudos apenas no filo Arthopoda $(n=10)$ e algumas espécies os artigos científicos não identificaram o localidade do parasito encontrado impossibilitando de registrar o continente (NI) apresentou registro de parasitos nos filos Platylhelmintes $(n=7)$, Arthopoda $(n=3)$. Percebe-se a importância dos trabalhos de revisão de literatura, pois facilita o trabalho de futuros pesquisadores em agilizar a pesquisa.
\end{abstract}

Palavras chave: Parasitismo, peixe sol, platelmintos, sunfish

\section{Check List of the parasites of the ocean sunfish (Mola mola: Molidae) fish in the world}

ABSTRACT. Due to the scarcity of works related to parasitism in the world, it is important to carry out a bibliographic review of scientific articles, dissertations and theses in databases and journals that are easily accessible to students and researchers about the occurrence of parasites in M. spring between the years of 1904 - 2017. A species of fish popularly known as moon fish (Mola mola: Molidae) has been found in every ocean in the world. Parasitism may be one of the next causes of family sweeping in Brazil, since there are a number of reduced scientific reports of occurrences of $M$. mola species. The studies made it possible to find parasitic occurrence in M. mola in the five phyla and in the five continents, of which there was a higher parasitic occurrence in Europe in the Platylhelminte $(\mathrm{n}=28)$, Arthopoda $(\mathrm{n}=15)$ and Nematoda $(\mathrm{n}=1)$, and in America the phyla Acanthocephala $(n=1)$, Platylhelmintes $(n=27)$ and Arthopoda $(n=15)$, Oceania 
Platylhelmintes $(\mathrm{n}=7)$, Nematoda Asia were recorded Platylhelminths $(\mathrm{n}=3)$ and Arthopoda $(n=5)$, in Africa only studies were registered in the phylum Arthopoda $(n=10)$ and some species the scientific articles did not identify the locality of the parasite found impossible to register the continent (NI) presented a record of parasites in the Platylhelmintes $(n=7)$, Arthopoda $(n=3)$. It is noticed the importance of the work of literature review, as it facilitates the work of future researchers in streamlining the research.

Keywords: Parasitism, platelmints, sunfish

\section{Lista de los parásitos del pescado Lua (Mola mola: Molidae) en el mundo}

RESUMEN. Debido a la escasez de trabajos relacionados con el parasitismo en el mundo, se percibe la importancia de realizar una revisión bibliográfica en artículos científicos, disertaciones y tesis en bancos de datos y periódicos de fácil acceso para los estudiantes e investigadores acerca de las ocurrencias de parásitos en M. primavera entre los años 1904 - 2017. La especie de pescado conocida popularmente como peces de luna (Mola mola: Molidae) se encuentran en todos los océanos del mundo. El parasitismo puede ser una de las posibles causas de encalladura de esta familia en Brasil, ya que existe un número de publicaciones científicas reducida de relatos de ocurrencia de la especie M. mola. Se han encontrado 51 trabajos relacionados con parásitos de la especie $M$. mola en el mundo, en el estudio se registraron 44 especies de parásitos y 9 especies son sinónimos, o sea, son parásitos conocidos por más de un nombre. En los cinco filos y en los cinco continentes, se observó un mayor registro de ocurrencia parasitaria en Europa en los filos Platylhelminte $(\mathrm{n}=28)$, Arthopoda $(\mathrm{n}=15)$ y Nematoda $(\mathrm{n}=1)$ y en América los filos Acanthocephala $(\mathrm{n}$ $=1)$, Platylhelmintes $(\mathrm{n}=27)$ y Arthopoda $(\mathrm{n}=15)$, Oceanía Platylhelmintes $(\mathrm{n}=7)$, Nematoda $(\mathrm{n}=1)$ y Arthopoda $(\mathrm{N}=5)$, en África se registraron estudios sólo en el filo Arthopoda $(n=10)$ y algunas especies los artículos científicos no identificaron la localidad del parásito encontrado imposibilitando registrar (NI) presentó registro de parásitos en los filos Platylhelmintes $(n=7)$, Arthopoda $(n=3)$. Se percibe la importancia de los trabajos de revisión de literatura, pues facilita el trabajo de futuros investigadores en agilizar la investigación.

Palabras clave: Parasitismo, pez sol, platelmintos, sunfish

\section{Introdução}

Os peixes da espécie Mola mola pertence a ordem Tetraodontiforme e a família Molidae (Ahuir-Baraja et al., 2017), conhecido popularmente como peixe Lua, peixe Sol ou molas. São encontrados nos oceanos Atlântico e Pacífico (Pope et al., 2010). O primeiro registro de ocorrência da espécie $M$. mola na costa brasileira capturado na Baía de Guanabará foi datado por Schreiner \& Ribeiro (1903).

A familia Molidae possui os maiores peixes teleósteos do mundo (Gustinelli et al., 2006). Os peixes são animais vertebrados que possui uma grande carga parasitária (Cavalcanti et al., 2012). Isso ocorre porque o meio aquático não apresenta uma temperatura estável, provocando a diminuição do metabolismo dos peixes, causando uma situação propicia para a proliferação parasitária (Andrade et al., 2001).
O parasitismo em peixes está associado a diversos fatores, como a dieta do hospedeiro e a idade, a composição química da água, a profundidade do hábitat, os fatores geográficos e as estações do ano (Pavanelli et al., 2013), levando em consideração a espécie $M$. mola composta de peixes migradores sazonais e migram em duas estações do ano, inverno e verão (Breen et al., 2017), habitando água profundas com até 600 metros (Dewar et al., 2010) e se alimentando de zooplâncton gelatinosos, algas, medusa, pequenos crustáceos e peixes (Breen et al., 2017).

Os parasitos atualmente são reconhecidos como importantes componentes na biodiversidade global (Cavalcanti et al., 2013), ajudando na compreensão da biologia, na sobrevivência, na estrutura da população do hospedeiro e no funcionamento do ecossistema, influenciando diretamente as populações de peixes pela mortalidade ou indiretamente na redução da 
fecundidade, alterações no comportamento, redução na velocidade de natação ou aumento do risco de predação do hospedeiro (Longshaw et al., 2010).

No estudo do parasitismo em peixes marinhos é importante levar em consideração o tamanho corporal do hospedeiro como um bom indicador de preferências das espécies parasitárias. Os maiores hospedeiros fornecem uma maior diversidade de nicho aos parasitos, consomem mais presas e possibilita ao parasito um ambiente propício para o seu desenvolvimento e reprodução parasitária (Pavanelli et al., 2013). O estudo realizou uma revisão bibliográfica em artigos científicos, dissertações e teses em bancos de dados e periódicos de fácil acesso acerca das ocorrências de parasitas em M. mola entre os anos de 1904 - 2017.

\section{Material e Métodos}

Para elabora a lista dos parasitos do peixe Lua Mola mola no mundo, os registros foram obtidos por meio de busca nas plataformas Scopus, SciELO, Elsevier, Web of Science, SpringerLink, no mecanismo de busca do Google Acadêmico e no Portal Periódicos CAPES/MEC, utilizando as palavras chave: Mola mola, Molidae, Teleósteos, Sunfishes.

Para elaboração dos dados da parasitofauna do peixe Lua (M. mola) foram copilados dados dos seguintes estudos: Montgomery (1957), Gibson \& Bray (1977), Love \& Moser (1983), Margolis \& Kabata (1996), Bray \& Gibson (1997), Diaz (2000), Dippenaar (2004), Whittington (2004), Gustinelli et al.(2006), Abe \& Sekiguchi (2012), Fernández et al. (2016), Kohn \& Fernandez (2016), Hogans (2017), onde estes autores citam outros trabalhos.

\section{Resultados e Discussão}

Foram encontrados 51 trabalhos relacionados a parasitos da espécie Mola mola no mundo, no estudo foram registrados 44 espécies de parasitos e 9 espécies são sinônimos, destes 2 espécies pertencem ao filo Acanthocephala há registro de apenas um parasito, com habitat não informado encontrado em Massachussetts (América do Norte), o filo Platelmintos está subdividido em três classes: a classe Monogenea foram registrados 3 espécies e 5 sinônimos, a classe Digenea foram registrados 17 espécies, a classe Cestoda foram registrados 5 espécies e um parasito apenas identificado a família apresentando características morfológicas diferentes com o Tipo I e II. O filo nematoda foi registrado em duas espécies e o filo Arthropoda 16 espécies e 4 sinônimos listrados na tabela 1.

Entre os anos de 1904 a 2017 foram encontrados estudos de ocorrência de parasitária em M. mola nos cinco filos e nos cinco continentes (Figura 1), destes houve maior registro de ocorrência parasitária na Europa nos filos Platylhelminte $(n=28)$, Arthopoda $(n=15)$ e Nematoda $(\mathrm{n}=1)$ e na América os filos Acanthocephala $(n=1)$, Platylhelmintes $(n=27)$ e Arthopoda $(\mathrm{n}=15)$, Oceania Platylhelmintes $(n=7)$, Nematoda $(n=1)$ e Arthopoda $(n=14) . \mathrm{Na}$ Ásia foram registrados os filos Platylhelmintes $(n=3)$ e Arthopoda $(n=5)$, na África foram registrados estudos apenas no filo Arthopoda $(n=10)$ e de algumas espécies os artigos científicos não identificaram a localidade do parasito encontrado impossibilitando registrar o continente (NI) apresentou registro de parasitos nos filos Platylhelmintes $(n=7)$, Arthopoda $(n=3)$.

Tabela 1. Lista de ocorrências de Parasitos do Peixe Lua (M. mola) registrados (1904 - 2017) no mundo. Habitando o: A (anus), B (boca), BR (brânquia), CO (Cavidade Oral), E (esôfago), ES (estomago), F (faringe), FI (fígado), IN (Intestino), $\mathrm{P}$ (pele), M (músculo), N (nadadeira), NI (Não informado), O (Opérculo), S (serosa intestino) T (Tegumento), TD (Trato digestório).

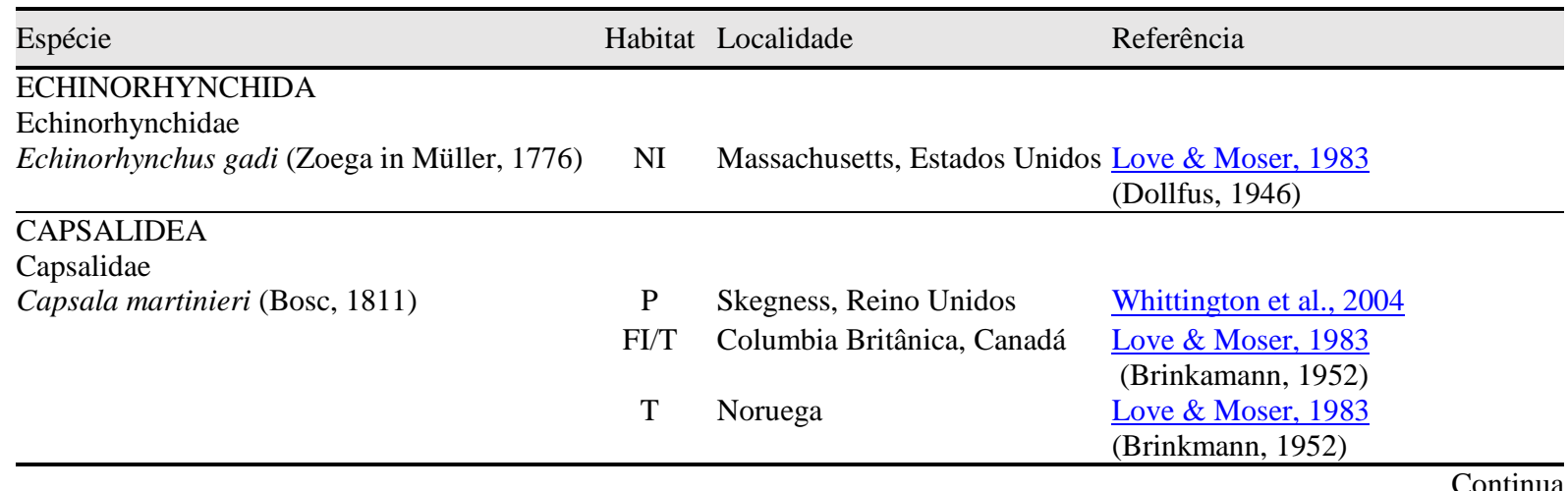




\begin{tabular}{|c|c|c|c|}
\hline Espécie & Habitat & Localidade & Referência \\
\hline$\overline{\text { Capsala martinieri (Bosc, } 1811)}$ & $\bar{T}$ & Terra Nova, Canadá & $\begin{array}{l}\text { Love \& Moser, } 1983 \\
\text { (Threlfall, 1967) }\end{array}$ \\
\hline \multirow[t]{2}{*}{ Capsala molae (Blanchard, 1847) (*) } & $\mathrm{T}$ & Massachussetts, EUA & $\frac{\text { Love \& Moser, } 1983}{\text { (Linton, 1940) }}$ \\
\hline & $\mathrm{T}$ & Nova Jersey, EUA & $\frac{\text { Love \& Moser, } 1983}{\text { (Prince, } 1962)}$ \\
\hline Capsala cephala (Riss0, 1826) (*) & NI & Europa & $\frac{\text { Love \& Moser, 1983 }}{\text { (Yamaguti, 1963) }}$ \\
\hline Capsala cutaneum (Guiart, 1938) (*) & NI & NI & $\frac{\text { Love \& Moser, } 1983}{\text { (Dawes, 1947) }}$ \\
\hline Capsala Grimaldi (Guiart, 1938) (*) & NI & NI & $\frac{\text { Love \& Moser, } 1983}{\text { (Dawes, 1947) }}$ \\
\hline Capsala muculata (Rudolphi) (*) & $\mathrm{T}$ & França & $\frac{\text { Love \& Moser, } 1983}{\text { (Dollfus, 1946) }}$ \\
\hline Capsala pelamydis (Taschenberg, 1878) & NI & NI & $\frac{\text { Love \& Moser, } 1983}{\text { (Dawes, 1947) }}$ \\
\hline Tristoma papillosum (Diesing, 1836) & $\mathrm{T}$ & França & $\frac{\text { Love \& Moser, } 1983}{\text { (Dollfus, 1946) }}$ \\
\hline \multicolumn{4}{|l|}{ PLAGIORCHIIDA } \\
\hline \multirow[t]{3}{*}{ Accacladium alveolatum } & IN & Atlântico & $\frac{\text { Love \& Moser, 1983 }}{\text { (Robinson, 1934) }}$ \\
\hline & IN & Nova Zelândia & $\frac{\text { Love \& Moser, } 1983}{\text { (Manter, 1960) }}$ \\
\hline & IN & Callao, Peru & $\frac{\text { Kohn \& Fernandes, } 2016}{\text { (Tantaleán et al., 1992) }}$ \\
\hline \multirow[t]{5}{*}{ Accacladium serpentulus (Odhner, 1928) } & IN & Califórnia, EUA & Gibson; Bray, 1977 \\
\hline & IN & Japão & $\frac{\text { Love \& Moser, } 1983}{\text { (Dawes, 1947) }}$ \\
\hline & $\mathrm{F}$ & Terra Nova, Canadá & $\frac{\text { Love \& Moser, } 1983}{\text { (Threlfall, 1967) }}$ \\
\hline & IN & Playa de Tomé, Chile & Fernández et al., 2016 \\
\hline & IN & Tomé, Chile & $\overline{\text { Fernández et al., } 2016}$ \\
\hline \multirow[t]{3}{*}{ Rhynchopharynx paradoxa (Odhner, 1928) } & IN & Japão & $\frac{\text { Love \& Moser, 1983 }}{\text { (Yamaguti, 1934) }}$ \\
\hline & & França & $\frac{\text { Love \& Moser, } 1983}{\text { (Dollfus, 1935) }}$ \\
\hline & & Nova Zelândia & $\frac{\text { Love \& Moser, } 1983}{\text { (Manter, 1960) }}$ \\
\hline \multirow[t]{5}{*}{ Accacoelium contortum (Rudolphi, 1819) } & FI & França & $\frac{\text { Love \& Moser, } 1983}{\text { (Linton, 1940) }}$ \\
\hline & $\mathrm{F}$ & Massachusetts, EUA & $\frac{\text { Love \& Moser, } 1983}{\text { (Threlfall, 1967) }}$ \\
\hline & & França & $\frac{\text { Love \& Moser, } 1983}{\text { (Timon-David; Musso, 1971) }}$ \\
\hline & $\mathrm{CO} / \mathrm{E}$ & Costa Italiana & Gustinelli et al.,2006 \\
\hline & IN & Nova Zelândia & $\frac{\overline{\text { Love \& Moser, } 1983}}{\text { (Manter, 1960) }}$ \\
\hline \multirow[t]{8}{*}{ Accacladocoelium macrocotyle (Diesing, 1858) } & IN & Massachusetts, EUA & $\frac{\text { Love \& Moser, } 1983}{\text { (Linton, 1940) }}$ \\
\hline & IN & Irlanda & $\frac{\text { Love \& Moser, } 1983}{\text { (Dawes, 1947) }}$ \\
\hline & IN & Naples, Florida & $\frac{\text { Love \& Moser, } 1983}{\text { (Dawes, 1947) }}$ \\
\hline & IN & Canadá & $\frac{\text { Love \& Moser, } 1983}{\text { (Dawes, 1947) }}$ \\
\hline & IN & Oregon, EUA & $\frac{\text { Love \& Moser, } 1983}{\text { (Pratt; Mccauley, 1961) }}$ \\
\hline & IN & Callao, Peru & $\frac{\text { Kohn \& Fernandes, } 2016}{\text { (Rivera; Sarmiento, 1992) }}$ \\
\hline & IN & Costa Italiana & Gustinelli et al., 2006 \\
\hline & IN & Tomé, Chile & $\overline{\text { Fernandez et al., } 2016}$ \\
\hline
\end{tabular}




\begin{tabular}{|c|c|c|c|}
\hline Espécie & Habitat & Localidade & Referência \\
\hline \multirow[t]{2}{*}{ Accaclodocoelium nigroflavum (Rudolphi, 1819) } & IN & Europa & $\begin{array}{l}\text { Love \& Moser, } 1983 \\
\text { (Dawes, 1947) }\end{array}$ \\
\hline & IN & Mediterrâneo & $\frac{\text { Love \& Moser, } 1983}{\text { (Dawes, 1947) }}$ \\
\hline \multirow[t]{5}{*}{ Odhnerium calyptrocotyle (Monticelli, 1893) } & IN & Japão & $\frac{\text { Love \& Moser, } 1983}{\text { (Yamaguti, 1934) }}$ \\
\hline & IN & Colúmbia Britânica, Canadá & $\frac{\text { Love \& Moser, } 1983}{\text { (Lloyd, 1938) }}$ \\
\hline & IN & Massachusetts, EUA & $\frac{\text { Love \& Moser, } 1983}{\text { (Linton, 1949) }}$ \\
\hline & IN & Nova Zelândia & $\frac{\text { Love \& Moser, } 1983}{\text { (Manter, 1954) }}$ \\
\hline & IN & Sul da Califórnia, EUA & $\underline{\text { Montgomery, } 1957}$ \\
\hline Orophocotyle planci (Stossich, 1899) & NI & Trieste, Itália & $\frac{\text { Love \& Moser, } 1983}{\text { (Dawes, 1947) }}$ \\
\hline \multicolumn{4}{|l|}{ Lepocreaddidae } \\
\hline \multirow[t]{9}{*}{ Dihemistephanus lydiae (Stossich, 1896) } & IN & $\begin{array}{l}\text { Staint Andrew Bay, Florida, } \\
\text { EUA }\end{array}$ & $\frac{\text { Bray; Gibson, } 1997}{\text { (Nicoll, 1909) }}$ \\
\hline & IN & $\begin{array}{l}\text { Firth of Fort, Escócia, Reino } \\
\text { Unido }\end{array}$ & $\frac{\mathrm{oBray} \text {; Gibson, } 1997}{\text { (Nicoll, 1915) }}$ \\
\hline & $\mathrm{TD}$ & França & $\frac{\text { Love \& Moser, } 1983}{\text { (Dollfus, 1946) }}$ \\
\hline & $\mathrm{TD}$ & Nova Zelândia & $\frac{\text { Love \& Moser, } 1983}{\text { (Manter, 1960) }}$ \\
\hline & IN & Nordeste do Oceano Atlântico & $\frac{\text { Margolis; Kabata, } 1996}{\text { (Dollfus, 1960) }}$ \\
\hline & IN & Saint-Vaast-la Hougue, França & $\frac{\text { Bray; Gibson, 1997 }}{\text { (Doullfus, 1960) }}$ \\
\hline & IN & Belle, Isle, França & $\frac{\text { Bray; Gibson, } 1997}{\text { (Doullfus, 1960) }}$ \\
\hline & IN & Nordeste do Golfo de Biscaia & $\frac{\text { Bray; Gibson, } 1997}{\text { (Doullfus, 1960) }}$ \\
\hline & IN & Whitby, Inglaterra & $\frac{\text { Bray; Gibson, } 1997}{\text { (Boxshall, 1977) }}$ \\
\hline \multirow[t]{2}{*}{ Dihemistephanus fragilis (Linton, 1900) } & $\mathrm{TD}$ & Massachusetts, EUA & $\frac{\text { Love \& Moser, } 1983}{\text { (Stafford, 1904) }}$ \\
\hline & $\mathrm{TD}$ & Terra Nova, Canadá & $\frac{\text { Love \& Moser, } 1983}{\text { (Threlfall, 1967) }}$ \\
\hline \multicolumn{4}{|l|}{ Macroderoididae } \\
\hline \multicolumn{4}{|l|}{ Acanthocolpidae } \\
\hline Stephanostomum baccatum (Nicoll, 1907) & NI & Massachusetts, EUA & $\frac{\text { Love \& Moser, } 1983}{\text { (Linton, 1940) }}$ \\
\hline \multicolumn{4}{|l|}{ Didymozoidae } \\
\hline Nematobibothrioides histoidii (Noble, 1974) & NI & Califórnia, EUA & $\frac{\text { Love \& Moser, } 1983}{\text { (Noble, 1975) }}$ \\
\hline Didymozoon molae (Rudolphi, 1819) & M & NI & $\frac{\text { Love \& Moser, } 1983}{\text { (Dollfus, 1946) }}$ \\
\hline Nematobothrium molae (Maclaren, 1904) & FI & NI & $\frac{\text { Love \& Moser, } 1983}{\text { (Dawes, 1947) }}$ \\
\hline Koellikeria filicollis (Rudolphi, 1819) & IN & $\mathrm{NI}$ & $\frac{\text { Love \& Moser, } 1983}{\text { (Nicoll, 1915) }}$ \\
\hline \multicolumn{4}{|l|}{$\begin{array}{l}\text { BOTHRIOCEPHAKIDAE } \\
\text { Triaenophoridae }\end{array}$} \\
\hline $\begin{array}{l}\text { Triaenophoridae } \\
\text { Tipo I }\end{array}$ & IN & Costa Italiana & $\underline{\text { Gustinelli et al., } 2006}$ \\
\hline $\begin{array}{l}\text { Triaenophoridae } \\
\text { Tipo II }\end{array}$ & IN & Costa Italiana & $\underline{\text { Gustinelli et al., } 2006}$ \\
\hline \multirow[t]{3}{*}{ Anchistrocephalus microcephalus (Rudolphi, 1819) } & IN & França & $\frac{\text { Love \& Moser, } 1983}{\text { (Dollfus, 1946) }}$ \\
\hline & IN & Nova Zelândia & $\frac{\text { Love \& Moser, } 1983}{\text { (Robinson, 1959) }}$ \\
\hline & & Terra Nova e Labrado, Canadá & $\frac{\text { Love \& Moser, } 1983}{\text { (Threlfall, 1967) }}$ \\
\hline
\end{tabular}




\begin{tabular}{|c|c|c|c|}
\hline Espécie & Habitat & Localidade & Referência \\
\hline & & Costa Italiana & Gustinelli et al., 2006 \\
\hline & & Playa de Tomé, Chile & Fernández et al., 2016 \\
\hline \multicolumn{4}{|l|}{ Pterobothriidae } \\
\hline Pterobothrium sp. & FI & Costa Italiana & Gustinelli et al., 2006 \\
\hline \multicolumn{4}{|l|}{ Trypanorhyncha } \\
\hline \multicolumn{4}{|l|}{ Gymnorhynchidae } \\
\hline \multirow[t]{3}{*}{ Molicola horridus } & NI & Nova Zelândia & $\frac{\text { Love \& Moser, } 1983}{\text { (Robinson, 1959) }}$ \\
\hline & $\mathrm{M} / \mathrm{FI}$ & Costa italiana & Gustinelli et al., 2006 \\
\hline & FI & Playa de Tomé, Chile & Fernández et al., 2016 \\
\hline \multicolumn{4}{|l|}{ Lacistorhynchidae } \\
\hline Floriceps saccatus (Cuvier, 1817) & $\mathrm{T}$ & França & $\frac{\text { Love \& Moser, } 1983}{\text { (Dollfus, 1946) }}$ \\
\hline Rhynchobothrium sp. (Larva) & I & Terra Nova, Canadá & $\frac{\text { Love \& Moser, } 1983}{\text { (Threlfall, 1967) }}$ \\
\hline \multirow[t]{2}{*}{ Anisakis sp. } & $\mathrm{V}$ & Nova Zelândia & Hewitt \& Hine, 1972 \\
\hline & $\mathrm{S}$ & Playa de Tomé, Chile & Fernández et al., 2016 \\
\hline \multicolumn{4}{|l|}{ Cucullanidae } \\
\hline Cucullanus orthagorisci & NI & França & $\frac{\text { Love \& Moser, } 1983}{\text { (Dollfus, 1946) }}$ \\
\hline \multicolumn{4}{|l|}{ SIPHONOSTOMATOIDA } \\
\hline \multirow[t]{7}{*}{ Pennella filosa (Linnaeus, 1758) } & NI & Oceano Pacifico & $\frac{\text { Love \& Moser, } 1983}{\text { (Wilson, 1932) }}$ \\
\hline & M & Massachusett, EUA & $\frac{\text { Love \& Moser, } 1983}{\text { (Wilson, 1932) }}$ \\
\hline & NI & França & $\frac{\text { Love \& Moser, } 1983}{\text { (Dollfus, 1946) }}$ \\
\hline & NI & África do Sul & $\frac{\text { Love \& Moser, } 1983}{\text { (Bernard, 1955) }}$ \\
\hline & NI & Table Bay, San Diego, EUA & $\frac{\text { Dippenaar, } 2004}{\text { (Oldewage, 1992) }}$ \\
\hline & $\mathrm{T}$ & Oeste do Oceano Pacifico & Abe; Sekiguchi, 2012 \\
\hline & $\mathrm{T}$ & Campobello, Irlanda & Hogans, 2017 \\
\hline Pennella crassicornis (Steenstrup \& Lutken, 1861) (*) & NI & NI & $\frac{\text { Love \& Moser, } 1983}{\text { (Yamaguti, 1963) }}$ \\
\hline \multicolumn{4}{|l|}{ Caligidae } \\
\hline \multirow[t]{5}{*}{ Lepeophtheirus nordmanni (Milne Edwards, 1840) } & $\mathrm{T}$ & França & $\frac{\text { Love \& Moser, } 1983}{\text { (Dollfus, 1946) }}$ \\
\hline & $\mathrm{T}$ & Nova Zelândia & $\frac{\text { Love \& Moser, } 1983}{\text { (Hewitt, 1964) }}$ \\
\hline & & $\begin{array}{l}\text { Cape Town, Cidade do Cabo } \\
\text { África do Sul }\end{array}$ & , \\
\hline & $\mathrm{P} / \mathrm{A}$ & Golfo de Cariaco, Venezuela & Diaz, 2000 \\
\hline & $\mathrm{P} / \mathrm{CO}$ & Costa Italiana & Gustinelli et al., 2006 \\
\hline \multirow[t]{5}{*}{ Lepeophtheirus insignis (Wilson C. B., 1908) $\left(^{*}\right)$} & $\mathrm{NI}$ & Sul da Califórnia, EUA & $\begin{array}{l}\text { Love \& Moser, } 1983 \\
\text { (Wilson, 1908) }\end{array}$ \\
\hline & NI & Table Bay, San Diego, EUA & $\frac{\text { Dippenaar, 2004 }}{\text { (Barnard, 1948) }}$ \\
\hline & NI & Japão & $\frac{\text { Love \& Moser, } 1983}{\text { (Shiino, 1957) }}$ \\
\hline & NI & Terra Nova, Canadá & $\frac{\text { Love E Moser, 1983 }}{\text { (Threlfall, 1967) }}$ \\
\hline & NI & Porto Elizabeth, África do Sul & $\frac{\text { Dippenaar, } 2004}{\text { (Kensley; Grindley, 1973) }}$ \\
\hline \multirow[t]{3}{*}{ Lepeophtheirus hastatus (Shiino, 1960) } & $\mathrm{T}$ & Japão & $\frac{\text { Love \& Moser, } 1983}{(\text { Shiino, 1960) }}$ \\
\hline & $\mathrm{T}$ & Austrália & $\frac{\text { Love \& Moser, } 1983}{\text { (Heegaard, 1962) }}$ \\
\hline & $\mathrm{T}$ & Nova Zelândia & $\frac{\text { Love \& Moser, } 1983}{\text { (Hewitt, 1964) }}$ \\
\hline
\end{tabular}




\begin{tabular}{|c|c|c|c|}
\hline Espécie & Habitat & Localidade & Referência \\
\hline Caligus elongatus (Von Nordmann, 1832) & NI & Norte do Mar Mediterrâneo & $\frac{\text { Love \& Moser, } 1983}{\text { (Parker, 1969) }}$ \\
\hline \multicolumn{4}{|l|}{ Lernaeopodidae } \\
\hline Lernaeopodoa bidiscalis (Kane, 1892) & $\mathrm{T}$ & França & $\frac{\text { Love \& Moser, } 1983}{\text { (Dollfus, 1946) }}$ \\
\hline \multicolumn{4}{|l|}{ Pandaridae } \\
\hline Pandarus bicolor (Leach, 1816) & $\mathrm{T}$ & França & $\frac{\text { Love \& Moser, } 1983}{\text { (Dollfus, 1946) }}$ \\
\hline \multicolumn{4}{|l|}{ Dichelesthiidae } \\
\hline Anthosoma crassum (Abildgaard, 1794) & $\mathrm{O} / \mathrm{N}$ & França & $\frac{\text { Love \& Moser, } 1983}{\text { (Dollfus, 1946) }}$ \\
\hline \multicolumn{4}{|l|}{ Trebiidae } \\
\hline Trebius sp. (Kroyer, 1837) & $\mathrm{T} / \mathrm{F}$ & França & $\frac{\text { Love \& Moser, } 1983}{\text { (Dollfus, 1946) }}$ \\
\hline \multicolumn{4}{|l|}{ Lepadidae } \\
\hline Conchoderma virgatum (Spengler, 1789) & $\mathrm{T}$ & NI & $\frac{\text { Love \& Moser, 1983 }}{\text { (Balakrishnan, 1969) }}$ \\
\hline \multicolumn{4}{|l|}{ Pandaridae } \\
\hline Echthrogaleus coleoptratus & $\mathrm{T}$ & França & $\frac{\text { Love \& Moser, } 1983}{\text { (Dollfus, 1946) }}$ \\
\hline \multirow[t]{7}{*}{ Cecrops latreillii (Leach, 1816) } & FI & França & $\frac{\text { Love \& Moser, } 1983}{\text { (Dollfus, 1946) }}$ \\
\hline & NI & $\begin{array}{l}\text { Cape Town, Cidade do Cabo } \\
\text { África do Sul }\end{array}$ & $\frac{\text { Dippenaar, 2004 }}{\text { (Bernard, 1955) }}$ \\
\hline & FI & Terra Nova, Canadá & $\frac{\text { Love \& Moser, } 1983}{\text { (Threlfall, 1967) }}$ \\
\hline & FI & Nova Zelândia & $\frac{\text { Love \& Moser, } 1983}{\text { (Hewitt, 1968) }}$ \\
\hline & BR & Golfo de Cariaco, Venezuela & Diaz, 2000 \\
\hline & $\mathrm{BR}$ & Costa Italiana & Gustinelli et al., 2006 \\
\hline & $\mathrm{BR}$ & Playa de Tomé, Chile & $\underline{\text { Fernández et al., } 2016}$ \\
\hline Cecrops exiguus (Wilson C.B., 1923) (*) & $\mathrm{CO} / \mathrm{FI}$ & Japão & $\frac{\text { Love \& Moser, } 1983}{\text { (Shiino, 1965) }}$ \\
\hline \multirow[t]{5}{*}{ Orthagoriscicola muricatus (Kroyer, 1837) } & $\mathrm{T} / \mathrm{FI}$ & França & $\frac{\text { Love \& Moser, } 1983}{\text { (Dollfus, 1946) }}$ \\
\hline & NI & África do Sul & $\frac{\text { Love \& Moser, } 1983}{\text { (Bernard 1955) }}$ \\
\hline & NI & Table Bay, San Diego, EUA & $\frac{\text { Dippenaar, 2004 }}{\text { (Bernard, 1955) }}$ \\
\hline & NI & Massachusetts, EUA & $\frac{\text { Love \& Moser, } 1983}{\text { (Wilson, 1970) }}$ \\
\hline & NI & Porto Elizabeth, África do Sul & $\frac{\text { Dippenaar, } 2004}{\text { (Kensley; Grindley, 1973) }}$ \\
\hline \multirow[t]{5}{*}{ Philorthagoriscus serratus (Kroyer, 1863) } & $\mathrm{T}$ & Massachusetts, EUA & $\frac{\text { Love \& Moser, } 1983}{\text { (Wilson, 1932) }}$ \\
\hline & $\mathrm{NI}$ & Table Bay, San Diego, EUA & $\frac{\text { Dippenaar, 2004 }}{\text { (Barnard, 1948) }}$ \\
\hline & $\mathrm{T}$ & África do Sul & $\frac{\text { Love \& Moser, } 1983}{\text { (Bernard, 1955) }}$ \\
\hline & $\mathrm{T}$ & Japão & $\frac{\text { Love \& Moser, } 1983}{\text { (Shiino, 1959) }}$ \\
\hline & $\mathrm{T}$ & Terra Nova, Canadá & $\frac{\text { Love \& Moser, } 1983}{\text { (Threlfall, 1967) }}$ \\
\hline \multirow[t]{2}{*}{ Dinematura latifólia (Steenstrup \& Lütken, 1861) } & $\mathrm{NI}$ & $\begin{array}{l}\text { Cape Town, Cidade do Cabo } \\
\text { África do Sul }\end{array}$ & 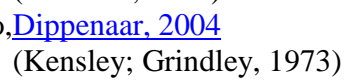 \\
\hline & NI & $\begin{array}{l}\text { Cape, Cidade do Cabo, Áfric } \\
\text { do Sul }\end{array}$ & aDippenaar, 2004 \\
\hline $\begin{array}{l}\text { DECAPODA } \\
\text { Epialtidae }\end{array}$ & & & \\
\hline \multirow[t]{2}{*}{ Acanthonyx scutiformis (Dana, 1851) } & $\mathrm{T}$ & França & $\frac{\text { Love \& Moser, } 1983}{\text { (Dollfus, 1946) }}$ \\
\hline & $\mathrm{T}$ & Japão & $\frac{\text { Love \& Moser, } 1983}{\text { (Yamaguti, 1963) }}$ \\
\hline
\end{tabular}




\begin{tabular}{|c|c|c|c|}
\hline Espécie & Habitat & Localidade & Referência \\
\hline \multicolumn{4}{|l|}{$\overline{\text { ISOPODA }}$} \\
\hline Nerocila orbignyi (Guérin-Méneville, 1832) & $\mathrm{T}$ & França & $\frac{\text { Love \& Moser, } 1983}{\text { (Dollfus, 1946) }}$ \\
\hline Nerocila maculata (H. Milne Edwars, 1840) (*) & $\mathrm{T}$ & França & $\frac{\text { Love \& Moser, } 1983}{\text { (Dollfus 1946) }}$ \\
\hline Ceratothoa steindachneri (Koelbel, 1879) & $\mathrm{CO}$ & Costa Italiana & Gustinelli et al., 2006 \\
\hline
\end{tabular}

(*) Sinônimo de uma espécie - Fonte: (Autora, 2018)

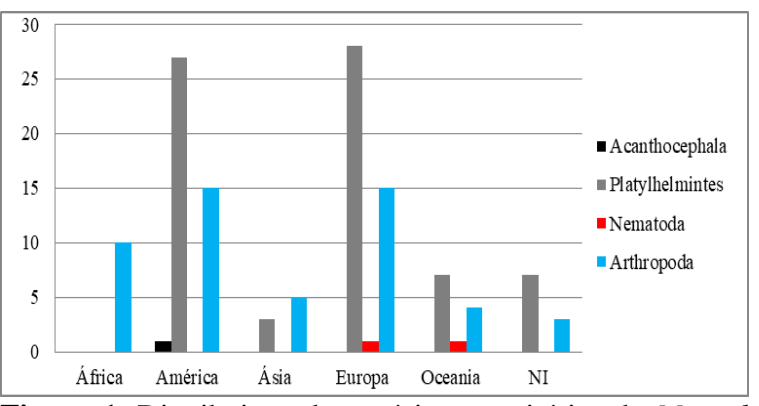

Figura 1. Distribuição de espécies parasitárias de $M$. mola nos continentes.

\section{Conclusão}

Percebe-se a importância dos trabalhos de revisão de literatura, pois facilita o trabalho de futuros pesquisadores, quando há um cheklist de uma determinada espécie. Durante a pesquisa compreende-se que são escassas as pesquisas no ramo da parasitologia e principalmente no Brasil há poucos relatos de trabalhos com parasitos em Peixe da espécie Mola mola, mesmo com alguns relatos de encalhe da espécie em algumas praias litorânea brasileira.

\section{Agradecimento}

A CAPES/MEC pela bolsa de mestrado concedida.

\section{Referências Bibliográficas}

Abe, T. \& Sekiguchi, K. 2012. Why does the ocean sunfish bask? Communicative \& Integrative Biology 5, 395-398

Ahuir-Baraja, A. E., Yamanoue, Y. \& Kubicek, L. 2017. First confirmed record of Mola sp. A in the western Mediterranean Sea: morphological, molecular and parasitological findings. Journal of Fish Biology, 90, 11331141.

Andrade, S. M. S., Malta, J. C. O. \& Ferraz, E. 2001. Fauna parasitológica de alevinos de matrinxã, Brycon cephalus (Günther, 1869) coletados nos rios Negro e Solimões, na
Amazônia Central. Acta Amazonica, 31, 263273.

Bray; R. A. \& Gibson, D. I. 1997. The Lepocreadiidae Odgner, 1905 (Digenea) of fishes from the north-east Atlantic: summary paper, with keys ans checklists. Systematic Parasitology 36, 223-228.

Breen, P., Cañadas, A., Cadhla, O. Ó., Mackey, M., Scheidat, M., Geelhoed, S. C. V., Rogan, E. \& Jessopp, M. 2017. New insights into ocean sunfish (Mola mola) abundance and seasonal distribution in the northeast Atlantic. Scientific Reports, 7, 1-9.

Cavalcanti, E. T. S., Nascimento, W. S., Takemoto, R. M., Alves, L. C. \& Chellappa, S. 2013. Ocorrência de crustáceos ectoparasitos no peixe ariacó, Lutjanus synagris (Linnaeus, 1758) nas águas costeiras do Rio Grande do Norte. Biota Amazônia, 3, 94-99.

Cavalcanti, E. T. S., Takemoto, R. M., Alves, L. C. \& Chellappa, S. 2012. First report of metazoan fish parasites with zoonotic potential in Scomberomorus brasiliensis and Trichiurus lepturus from the coastal waters of Rio Grande do Norte, Brazil. Marine Biodiversity Records, 5, e40.

Dewar, H., Thys, T., Teo, S. L. H., Farwell, C., O'Sullivan, J., Tobayama, T., Soichi, M., Nakatsubo, T., Kondo, Y. \& Okada, Y. 2010. Satellite tracking the world's largest jelly predator, the ocean sunfish, Mola mola, in the Western Pacific. Journal of Experimental Marine Biology and Ecology, 393, 32-42.

Diaz, O. D. 2000. Copépodos ectoparasitos del Pez Luna Mola mola (Giglioli, 1883) (Pisces: Molidae) em el Golfo de Cariaco, Venezuaela. Venezuela. Boletín del Instituto Oceanográfico, Venezuela, Universidad Oriente 39, 11-17.

Dippenaar, S. M. 2004. Reported siphonostomatoid copepods parasitic on marine fishes of southern Africa. Crustaceana 77, 1281-1328. 
Fernández, I., Oyarzún, C., Valenzuela, Ariel., Burgos, C., Guaquín, V. \& Campos, V. 2016. Parásitos del pez luna Mola mola (Pisces: Molidae). Primer registro em aguas de la cosra centro sur de Chile. Gayana 80, 192-197.

Gibson, D. I. \& Bray, R. A. 1977. The Azygiidae, Hirudinellidae, Ptychogonimidae, Sclerodistomidae and Syncoeliidae (Digenea) of fishes from the northeast Atlantic. Bulletin of the British Museum, Natural History. Zoology, 32, 167-245.

Gustinelli, A., Nardini, G., Aureli, G., Trentini, M., Affronte, M. \& Fioravanti, M. L. 2006. Parassitofauna di Mola mola (Linnaeus, 1758) dei mari italiani. Biologia Marina Mediterranea, 13, 872-876.

Hogans, W. W. 2017. Review of Pennella Oken, 1816 (Copepoda: Pennellidae) with a description of Pennella benzi sp. Nov., a parasite of Escolar, Lepidocybium flavobrunneum (Pisces) in the northwest Atlantic Ocean. Zootaxa 4244 001-038.

Kohn, A., Fernandes, B. M. M. 2016. Trematoda, IN: Eiras, J. C.; Velosso, A. L. \& Pereira JR, J. Parasito de peixes marinhos da América do Sul [Recursos eletrônico] - Rio Grande: ed. Da FURG.pp. 95-169.

Longshaw, M., Frear, P. A., Nunn, A. D., Cowx, I. G. \& Feist, S. W. 2010. The influence of parasitism on fish population success. Fisheries Management and Ecology, 17, 426434.

Love, S. M. \& Moser, M. 1983. A Checklist of Parasites of California, Oregon, and Washington Marine and Estuarine Fishes. Faculty Publications from the Harold W. Manter Laboratory of Parasitology. Paper 750.

Margolis, L. \& Kabata, Z. 1996. Guide to the parasites of Fishes of Canada. Part IV Trematoda (By Gibson, D. I.). Canadian
Special Publication of Fisheries ans Aquatic Sciences 124, NCR Research Press, Ottawa.

Montgomery, W. R. 1957. Studies on Digenetic Trematodes from Marine Fishes of La Jolla, California. Transactions of the American Microscopical Society.76, 13-36.

Pavanelli, G. C., Takemoto, R. M. \& Eiras, J. C. 2013. Parasitologia de peixes de água doce do Brasil. EDUEM, Maringá, Paraná.

Pope, E. C., Hays, G. C., Thys, T. M., Doyle, T. K., Sims, D. W., Queiroz, N., Hobson, V. J., Kubicek, L. \& Houghton, J. D. R. 2010. The biology and ecology of the ocean sunfish Mola mola: a review of current knowledge and future research perspectives. Reviews in Fish Biology and Fisheries, 20, 471-487.

Schreiner, C. \& Ribeiro, A. M. 1903. A collecção de peixes do Museu Nacional do Rio de Janeiro. Archivos do Museu Nacional do Rio de Janeiro, 12, 67-109.

Whittington I. D., Deveney, M. R., Morgan, J. A. T. \& Chisholm, L. A. 2004. A preliminary phylogenetic analysis of the Capsalidae (Platyhelminthes: Monogenea: Monopisthocotylea) inferred from large subunit rDNA sequences. Parasitology 128: 511-519.

Article History:

Received 27 October 2017

Accepted 28 November 2017

Available online 15 January 2018

License information: This is an open-access article distributed under the terms of the Creative Commons Attribution License 4.0, which permits unrestricted use, distribution, and reproduction in any medium, provided the original work is properly cited. 